\title{
CANONICAL DECOMPOSITION OF HARMONIZABLE ISOTROPIC RANDOM CURRENTS
}

\author{
By YUKINAO ISOKAWA
}

\begin{abstract}
First we show that the difference between harmonizable isotropic random currents and homogeneous isotropic ones is small in some sense. Next we obtain the quasi canonical decomposition of harmonizable isotropic random currents, and discuss the possibility of the canonical decomposition of them. Then we show that a certain kind of harmonizable isotropic random currents which is not homogeneous has the canonical decomposition.
\end{abstract}

\section{$\S 1$. Introduction}

Let $\Omega_{p}$ be a class of random $p$-currents. We say that a random current $U_{p}$ has the quasi canonical decomposition in $\mathfrak{R}_{p}$ if it has the unique decomposition $U_{p}=U_{p}^{h}+U_{p}^{i}+U_{p}^{s}$ in $\Omega_{p}$ such that $d U_{p}^{i}=0, \quad \delta U_{p}^{s}=0$ and $\Delta U_{p}^{h}=0$. The random currents $U_{p}^{i}, U_{p}^{s}$ and $U_{p}^{h}$ are called the irrotational, the solenoidal and the harmonic components of $U_{p}$ respectively. If the covariances between any two components are zero in the quasi canonical decomposition, we call it the canonical decomposition. Physically the quasi canonical decomposition means the decomposition of a wave into the longitudinal one and the transversal one, and the canonical decomposition corresponds to the case where these two kinds of waves are stochastically independent.

Let $\mathfrak{H}_{p}$ be the class of homogeneous isotropic random $p$-currents, and $\mathfrak{W}_{p}$ be the class of harmonizable isotropic random $p$-currents. K. Ito (1956) has shown that every random current in $\mathfrak{H}_{p}$ has the canonical decomposition in it. In this paper we investigate the possibility of the canonical decomposition in $\mathfrak{B}_{p}$.

In Theorem 1 of $\S 2$ we have two characterizations of the class $\mathfrak{u}_{p}$ in the broader class $\mathfrak{W}_{p}$. The results may be understood as those stating that the difference between two classes is not large. Then we introduce a class $\mathfrak{V}_{p}$ of isotropic random currents which are superpositions of independent plane waves. This class stands between $\mathfrak{H}_{p}$ and $\mathfrak{B}_{p}$.

In $\S 3$ we first show that every random current in $\mathfrak{W}_{p}$ has the quasi canonical decomposition, but it is not necessarily the canonical one. Next we show

Received February 9, 1981 
that the canonical decomposition is possible in $\mathfrak{B}_{p}$. This means that we can not characterize $\mathfrak{H}_{p}$ by using the possibility of the canonical decomposition.

\section{$\S 2$. Relations among classes $\mathfrak{H}_{p}, \mathfrak{B}_{p}$ and $\mathfrak{M}_{p}$}

Let $L$ be the Hilbert space of random variables with mean 0 and finite variance. In $L$ the inner product $\langle$,$\rangle of two elements is defined by their$ covariance. Let $\mathscr{D}_{p}$ be the linear space of smooth $p$-vector fields with compact support, where we introduce the Schwartz topology. A random $p$-current $U_{p}$ is defined as a continuous linear map

$$
U_{p}: \mathfrak{D}_{n-p} \ni \phi_{n-p} \rightarrow U_{p}\left(\phi_{n-p}\right) \in L .
$$

For a random $p$-current $U_{p}$ and a constant $p$-vector $a_{p}$, we define $\left(U_{p}, a_{p}\right)$ as a random distribution, $\left(U_{p}, a_{p}\right)(\phi)=U_{p}\left(\phi^{*} a_{p}\right)$. The function defined by

$$
\rho\left(\phi, \phi ; a_{p}, b_{p}\right)=\left\langle\left(U_{p}, a_{p}\right)(\phi),\left(U_{p}, b_{p}\right)(\phi)\right\rangle
$$

is called the covariance bilinear form of $U_{p}$. For a $p$-vector field $\phi_{p} \in \mathscr{D}_{p}$ and a random $p$-current $U_{p}$, a motion $g: R^{n} \ni x \rightarrow g x \in R^{n}$ induces a new $p$-vector field $g \phi_{p}$ and a new random $p$-current $g U_{p}$ as

$$
\left(g \phi_{p}\right)(x)=g \phi_{p}\left(g^{-1} x\right) \text { and }\left(g U_{p}\right)\left(\phi_{n-p}\right)=U_{p}\left(g \phi_{n-p}\right) .
$$

If the covariance bilinear form of $g U_{p}$ does not depend on all translations $g$ or all rotations $g$, then $U_{p}$ is said to be homogeneous or isotropic respectively. A random current $U_{p}$ is said to be isotropic about the point $h$ if the translated random current $h U_{p}$ is isotropic. We say that a random current is harmonizable and its spectral measures are $m\left(d \lambda, d \mu ; a_{p}, b_{p}\right)$ if its its covariance bilinear form is written as

$$
\rho\left(\phi, \phi ; a_{p}, b_{p}\right)=\int_{R^{n} \times R^{n}} \mathscr{I} \phi(\lambda) \overline{\mathscr{I} \phi(\mu)} m\left(d \lambda, d \mu ; a_{p}, b_{p}\right),
$$

where $m\left(d \lambda, d \mu ; a_{p}, b_{p}\right)$ is a complex-valued tempered measure for each pair of constant $p$-vectors $a_{p}, b_{p}$, and $\mathscr{I} \phi$ denotes the Fourier transform of a function $\phi$,

$$
\mathscr{F} \phi(\lambda)=\int_{R^{n}} \exp (i(x, \lambda)) \phi(x) d x .
$$

A random measure is defined as a random current whose covariance bilinear form is of the form

$$
\rho\left(\phi, \phi ; a_{p}, b_{p}\right)=\int_{R^{n} \times R^{n}} \phi(\lambda) \overline{\psi(\mu)} m\left(d \lambda, d \mu ; a_{p}, b_{p}\right),
$$

where $m$ is a complex-valued tempered measure. In particular, if the support of $m$ is contained in the diagonal set $\left\{(\lambda, \mu) \in R^{n} \times R^{n} ; \lambda=\mu\right\}$ for every $a_{p}, b_{p}$, it is called an orthogonal random measure. 
In the below we frequently use the following lemma, which can be proved as in the proof of Proposition 5.1 of M. M. Rao (1969).

LEMMA 1. A harmonizable random current with spectral measures $m(d \lambda, d \mu$; $\left.a_{p}, b_{p}\right)$ is isotropic if and only if $m\left(d(g \lambda), d(g \mu) ; g a_{p}, g b_{p}\right)=m\left(d \lambda, d \mu ; a_{p}, b_{p}\right)$ hold for all rotations $g$ and all $a_{p}$ and $b_{p}$.

K. Ito has shown that a homogeneous random current is the Fourier transform of an orthogonal random measure. Applying Theorem 3.1 of M. M. Rao, we have similar representations for harmonizable random currents. We denote the Fourier transform of $\phi_{n-p} \in \mathfrak{D}_{n-p}$ by $\mathscr{I} \phi_{n-p}$.

Proposition 1. A harmonzable random current $U_{p}$ can be represented by a random measure $M_{p}$ as its Fourier transform, $U_{p}\left(\phi_{n-p}\right)=M_{p}\left(\mathscr{I} \phi_{n-p}\right)$. Conversely the random current defined by the Fourier transform of a random measure is harmonizable.

It is obvious from Proposition 1 that $\mathfrak{H}_{p}$ is contained in $\mathfrak{W}_{p}$. The following theorem states that the difference between these two classes is not so large as was expected by the comparison of their spectral measures.

THEOREM 1. A harmonizable random current in $\mathfrak{B}_{p}$ which is isotropic about the point $h \neq 0$ is homogeneous. Similarly, a random current $U_{p}$ in $\mathfrak{W}_{p}$ whose covarnance bilinear form conncides with that of $h U_{p}$ for a translation $h$ is homogeneous.

Proof. We only prove the first assertion as the proof of the second is similar. If $U_{p}$ has the spectral measures $m\left(d \lambda, d \mu ; a_{p}, b_{p}\right), h U_{p}$ has the spectral measure $\exp (i(\lambda-\mu, h)) m\left(d \lambda, d \mu ; a_{p}, b_{p}\right)$. Using Lemma 1, we have

$$
\left\{1-\exp \left(i\left(\lambda-\mu, h-g^{-1} h\right)\right)\right\} m\left(d \lambda, d \mu ; a_{p}, b_{p}\right)=0
$$

for all $a_{p}, b_{p}, \lambda, \mu$ and $g$. Throughout the argument we fix $a_{p}$ and $b_{p}$. For any $g$, there is a null set $N_{g}$ with respect to the measure $m\left(d \lambda, d \mu ; a_{p}, b_{p}\right)$ such that

$$
\exp (i((\lambda-\mu)-g(\lambda-\mu), h))=1
$$

for all $(\lambda, \mu) \notin N_{g}$. Take a countable dense subset $C$ of the orthogonal group $O(n)$, and put $N=\bigcup_{g \in C} N_{g}$. Choose any element $(\lambda, \mu) \notin N$, and fix it. Then, noting the continuity, we see that (1) hold for all $g \in O(n)$. We define $F=\{(\lambda-\mu)-g(\lambda-\mu) ; g \in O(n)\}$ and $P=\left\{x \in R^{n} ; x\right.$ is orthogonal to $\left.h\right\}$. Since $F$ is connected, $F$ is contained in $P$. If $\lambda-\mu \neq 0$, we have $n=\operatorname{dim} F \leqq \operatorname{dim} P=$ $n-1$. This is a contradiction. Accordingly the complement of the diagonal set is contained in $N$. Since $N$ is a null set, the measure $m\left(d \lambda, d \mu ; a_{p}, b_{p}\right)$ is concentrated on the diagonal set. Therefore $U_{p}$ is homogeneous. 
We introduce a class of random $p$-currents which may be understood as the superpositions of independent plane waves. Let $h$ be a complex-valued function on $R^{1}$ which is expressed as

$$
h(y)=\int_{R^{1}} \exp (i \alpha y) H(d \alpha)
$$

by a complex-valued measure $H$ such that the integrals

$$
\int_{|\alpha| \leqslant 1}|\alpha|^{-N}|H(d \alpha)|, \int_{|\alpha|>1}|\alpha|^{N}|H(d \alpha)|
$$

are finite for all $N \geqq 0$. Let $M_{p}$ be an orthogonal isotropic random measure on $R^{n}$ by which complex-valued tempered measures $m$ are defined as

$$
m\left(d t ; a_{p}, b_{p}\right)=\left\langle\left(M_{p}(d t), a_{p}\right),\left(M_{p}(d t), b_{p}\right)\right\rangle .
$$

We can prove easily that the map $\mathfrak{D}_{n-p} \ni \phi_{n-p} \rightarrow \mathfrak{S} \phi_{n-p} \in \mathfrak{D}_{n-p}$ is continuous, where

$$
\left(\mathfrak{S} \phi_{n-p}\right)(t)=\int_{R^{n}} h((x, t)) \phi_{n-p}(x) d x .
$$

Accordingly we can define a random current $U_{p}$ as the $\mathfrak{S}$-transform of $M_{p}$, $U_{p}\left(\phi_{n-p}\right)=M_{p}\left(\mathfrak{S} \phi_{n-p}\right)$. Now we define a class $\mathfrak{V}_{p}$ as the totality of the $\mathfrak{S}$-transform of orthogonal isotropic random $p$-measures for any $h$.

PROPOSITION 2. $\mathfrak{H}_{p} \subset \mathfrak{B}_{p} \subset \mathfrak{W}_{p}$

Proof. It is obvious that $\mathfrak{B}_{p}$ contains $\mathfrak{U}_{p}$. Consider a random current $U_{p}$ in $\mathfrak{B}_{p}$. From the assumption on $h$ it follows that $\mathfrak{S} \phi$ is a rapidly decreasing infinitely differentiable function for any $\phi \in \mathfrak{D}$. Thus we can write the covariance bilinear form of $U_{p}$ as

$$
\rho\left(\phi, \phi ; a_{p}, b_{p}\right)=\int_{R^{n}}(\mathfrak{S} \phi)(t) \overline{(\mathfrak{S} \phi)(t)} m\left(d t ; a_{p}, b_{p}\right) .
$$

From this expression we can see easily that $U_{p}$ is isotropic. Now we define $\Lambda(t)=\left\{\alpha \in R^{1} ; \alpha t \in \Lambda\right\}$ for any bounded Borel set $\Lambda$ in $R^{n}$, and

$$
z\left(\Lambda_{1}, \Lambda_{2} ; a_{p}, b_{p}\right)=\int_{t \neq 0} H\left(\Lambda_{1}(t)\right) \overline{H\left(\Lambda_{2}(t)\right)} m\left(d t ; a_{p}, b_{p}\right)
$$

for any bounded Borel set $\Lambda_{1}$ and $\Lambda_{2}$. Noting that

$$
(\mathfrak{S} \phi)(t)=\int_{R^{1}}(\widetilde{\mho} \phi)(\alpha t) H(d \alpha) \text {, }
$$

we can rewrite the covariance bilinear form as

$$
z\left(\phi, \phi ; a_{p}, b_{p}\right)=\int_{R^{n} \times R^{n}}(\mathfrak{F} \phi)(\lambda) \overline{(\widetilde{F} \phi)(\mu)} z\left(d \lambda, d \mu ; a_{p}, b_{p}\right) .
$$


Moreover we can prove that $z$ is a tempered measure. Therefore $U_{p}$ belongs to $\mathfrak{B}_{p}$.

\section{$\S$ 3. Canonical decomposition in classes $\mathfrak{H}_{p}, \mathfrak{B}_{p}$ and $\mathfrak{W}_{p}$}

First we consider the quasi canonical decomposition of harmonizable random currents.

PROPOSITION 3. Every harmonizable random current has the quasi canonical decomposition in which all components are harmonizable.

Proof. Let $U_{p}$ be a harmonizable random current which is the Fourier transform of $M_{p}$. We define four random measures $M_{p}^{h}, M_{p}^{u}, M_{p}^{i}$ and $M_{p}^{s}$ as

$$
\begin{array}{cl}
M_{p}^{h}(d \lambda)=M_{p}(d \lambda \cap\{0\}), & M_{p}^{u}(d \lambda)=M_{p}(d \lambda-\{0\}), \\
M_{p}^{i}(d \lambda)=|\lambda|^{-2} \lambda \wedge\left(\lambda \vee M_{p}^{u}(d \lambda)\right), & M_{p}^{s}(d \lambda)=|\lambda|^{-2} \lambda \vee\left(\lambda \wedge M_{p}^{u}(d \lambda)\right) .
\end{array}
$$

Moreover we define three random currents $U_{p}^{h}, U_{p}^{i}$ and $U_{p}^{s}$ as the Fourier transform of $M_{p}^{h}, M_{p}^{i}$ and $M_{p}^{s}$ respectively. Then, as in the proof of Theorem 5.2 of $\mathrm{K}$. Ito, we can show that $U_{p}$ has the quasi canonical decomposition.

Now we investigate the possibility of the canonical decomposition in $\mathfrak{W}_{p}$. We put $D=\left\{(\lambda, \mu) \in R^{n} \times R^{n} ; \lambda\right.$ and $\mu$ are linearly independent $\}$, and for each $(\lambda, \mu) \in D$, we choose a system of vectors $\left\{\xi=|\lambda|^{-1} \lambda, \eta=|\mu|^{-1} \mu, \zeta_{\jmath}(1 \leqq j \leqq n-2)\right\}$ such that $\zeta$ 's are orthonormal and orthogonal to both $\xi$ and $\eta$. Then, defining a measure $\tilde{m}$ in the domain $D$ as

$$
\tilde{m}(d \lambda, d \mu)=m\left(d \lambda, d \mu ; \xi \wedge \zeta_{1} \wedge \zeta_{2} \wedge \cdots \wedge \zeta_{p-1}, \frac{\xi-(\xi, \eta) \eta}{\left(1-(\xi, \eta)^{2}\right)^{1 / 2}} \wedge \zeta_{1} \wedge \cdots \wedge \zeta_{p-1}\right),
$$

we have the following theorem.

THEOREM 2. Every random current $U_{p}$ in $\mathfrak{W}_{p}$ has the quasi canonical decomposition in it. Its irrotational component and solenordal component are mutually orthogonal if and only if the measure $\tilde{m}$ is identically zero in the domain $D$.

Proof. From Proposition 3, $U_{p}$ has the quasi canonical decomposition $U_{p}=$ $U_{p}^{h}+U_{p}^{i}+U_{p}^{s}$ where all components are harmonizable. We have to show that they are also isotropic. We only prove for $U_{p}^{i}$. Noting the identity $\left(\lambda \wedge\left(\lambda \vee a_{p}\right), b_{p}\right)$ $=\left(a_{p}, \lambda \wedge\left(\lambda \vee b_{p}\right)\right)$, we have the following expression

$$
m^{\imath}\left(d \lambda, d \mu ; a_{p}, b_{p}\right)=m^{u}\left(d \lambda, d \mu ; \xi \wedge\left(\xi \vee a_{p}\right), \eta \wedge\left(\eta \vee b_{p}\right)\right)
$$

for spectral measures of $U_{p}^{i}$. Then, using Lemma 1, we can see that $U_{p}^{i}$ is isotropic.

Similarly cross-spectral measures of $U_{p}^{i}$ and $U_{p}^{s}$ can be written as

$$
m^{\imath s}\left(d \lambda, d \mu ; a_{p}, b_{p}\right)=m^{u}\left(d \lambda, d \mu ; \xi \wedge\left(\xi \vee a_{p}\right), \eta \vee\left(\eta \wedge b_{p}\right)\right)
$$


By the same reasoning as in the proof of Theorem 7.5 of I. Kubo (1967), we can rewrite them as

$$
m^{\imath s}\left(d \lambda, d \mu ; a_{p}, b_{p}\right)=\frac{1}{\left(1-(\xi, \eta)^{2}\right)^{1 / 2}}\left(\xi \wedge\left(\xi \vee a_{p}\right), \eta \vee\left(\eta \wedge b_{p}\right)\right) \tilde{m}(d \lambda, d \mu)
$$

in the domain $D$.

From this expression we derive heuristically that $m^{2 s}$ is identically zero in the complement of $D$ although we can prove it rigorously by the same reasoning as in the above. In fact, we see easily that the coefficient of $\tilde{m}$ in the above expression is bounded in $\xi$ and $\eta$, and moreover, when $\eta$ tends to $\xi$ or $(-\xi), \xi-(\xi, \eta) \eta$ is nearly perpendicular to $\xi$ and then $\tilde{m}(d \lambda, d \mu)$ is nearly equal to zero. Thus the proof is completed.

Every random current in $\mathfrak{S}_{p}$ does not have the canonical decomposition, while every random current in $\mathfrak{H}_{p}$ has it. On the other hand, Theorem 1 states that the difference between $\mathfrak{H}_{p}$ and $\mathfrak{W}_{p}$ is not large. Thus one may ask whether a random current in $\mathfrak{W}_{p}$ which has the canonical decomposition is homogeneous or not. The following theorem answers this question in the negative way.

THEOREM 3. Every random current in $\mathfrak{B}_{p}$ has the canonical decomposition in it.

Proof. The proof of the quasi canonical decomposition of random currents in $\mathfrak{B}_{p}$ is similar to that of harmonizable random currents in Proposition 3. We can see easily the orthogonality of the harmonic component and the other two ones. In the below we show that the irrotational component and the solenoidal component are mutually orthogonal. For any bounded Borel set $\Lambda$ not containing 0 , we put $c(\Lambda)=\left\{t \in R^{n}\right.$; there is $\alpha \in R^{1}$ and $\lambda \in R^{n}$ such that $\left.t=\alpha \lambda\right\}$. Using the notation in the proof of Proposition 2 , we see that $z\left(\Lambda_{1}, \Lambda_{2} ; a_{p}, b_{p}\right)$ is zero for any bounded Borel sets $\Lambda_{1}$ and $\Lambda_{2}$ such that $c\left(\Lambda_{1}\right) \cap c\left(\Lambda_{2}\right)=\{0\}$. Accordingly, by Theorem 2, we have the conclusion.

Acknowledgement-The author sincerely thanks Professor I. Kubo (Nagoya University) for his greatly valuable comments.

\section{REFERENCES}

[1] Iто, K., Isotropic random currents, in the Proceedings of the third Berkley Symposium on Mathematical Statistics and Probability, Berkley and Los Angels, University of Calfolnıa, Vol. 2, 125-132, 1956.

[2] Kubo, I., The topics in random fields, Seminar on Probability, Vol. 26, 1967 (Japanese).

[3] RAo, M. M., Representation theory of multidimensional generalized random fields, in the Proceedings of the Second International Symposium on Multivariate Analysis, Academic Press, New York, 411-436, 1965.

Department of Mathematics

TOKyo Institute of TECHNOLOgy 